\title{
Cost Effectiveness of Secukinumab for the Treatment of Active Psoriatic Arthritis in the UK
}

\author{
Vanessa Buchanan ${ }^{1}$ Will Sullivan ${ }^{1}$ - Chris Graham ${ }^{2} \cdot$ LaStella Miles $^{2}$. \\ Steffen Marc $\mathrm{Jugl}^{3}$ • Praveen Gunda ${ }^{4} \cdot$ Anna Halliday $^{5}$ - Bruce Kirkham ${ }^{6,7}$
}

Published online: 25 May 2018

(C) The Author(s) 2018

\begin{abstract}
Objective The aim was to determine the cost effectiveness of secukinumab, a fully human interleukin-17A inhibitor, for adults in the UK with active psoriatic arthritis (PsA) who are tumour necrosis factor inhibitor (TNFi) naïve and without concomitant moderate-to-severe psoriasis, and who have responded inadequately to conventional systemic disease-modifying anti-rheumatic drugs (csDMARDs). Perspective and setting The study took the perspective and setting of the UK National Health Service (NHS).
\end{abstract}

Vanessa Buchanan is formerly of BresMed Health Solutions and was affiliated with this organisation at the time of study.

Electronic supplementary material The online version of this article (https://doi.org/10.1007/s40273-018-0674-x) contains supplementary material, which is available to authorized users.

Anna Halliday

anna.halliday@novartis.com

BresMed Health Solutions, Sheffield, UK

2 RTI Health Solutions, Research Triangle Park, NC, USA

3 Novartis Pharma AG, Basel, Switzerland

4 Novartis Healthcare Private Limited, Hyderabad, India

5 Novartis Pharmaceuticals UK Limited, Camberley, UK

6 Guy's and St Thomas' NHS Foundation Trust, London, UK

7 King's College London, London, UK
Methods The model structure was a 3-month decision tree leading into a Markov model. Separate analyses based on the number of prior csDMARDs (one and two or more) were conducted, with secukinumab $150 \mathrm{mg}$ compared to standard of care (SoC) and TNFis, respectively, for each subpopulation. Clinical parameters, including response at 3 months, were from the FUTURE 2 trial and a network meta-analysis. Outcomes included total costs and qualityadjusted life years (QALYs) over the 40-year time horizon (3.5\% annual discount for both outcomes; cost year 2017), and incremental cost effectiveness ratios (ICERs).

Results The ICER for secukinumab $150 \mathrm{mg}$ versus SoC was $£ 28,748$ per QALY gained (one prior csDMARD). Secukinumab $150 \mathrm{mg}$ dominated golimumab, certolizumab pegol and etanercept, and had an ICER of $£ 5680$ per QALY gained versus adalimumab and $>£ 1$ million saved per QALY foregone versus infliximab (two or more prior csDMARDs). Valuing one QALY at between $£ 20,000$ and $£ 30,000$, the probability of secukinumab having the highest net monetary benefit was $48.9 \%$ (one prior csDMARD) and $88.9 \%$ (two or more prior csDMARDs). Parameters related to Health Assessment Questionnaire scores were most influential.

Conclusions Secukinumab $150 \mathrm{mg}$ at list price appears to represent a cost-effective use of NHS resources for adults with PsA who have responded inadequately to one or two or more prior csDMARDs. 


\section{Key Points for Decision Makers}

Secukinumab $150 \mathrm{mg}$ at list price may represent a cost-effective treatment option from the perspective of the UK National Health Service for patients with active psoriatic arthritis who are tumour necrosis factor inhibitor naïve, do not have concomitant moderate-to-severe psoriasis and who have responded inadequately to previous conventional systemic disease-modifying anti-rheumatic drugs (csDMARDs).

Secukinumab $150 \mathrm{mg}$ was associated with an incremental cost effectiveness ratio of less than $£ 30,000$ per quality-adjusted life year gained versus standard of care in the subpopulation of patients who responded inadequately to one prior csDMARD, and in some cases dominated tumour necrosis factor inhibitors in the two or more prior csDMARD subpopulation.

The availability of published subgroup data for all comparators would reduce uncertainty in cost effectiveness analyses of biologics at different stages of the treatment pathway in psoriatic arthritis. In the absence of such data for this evaluation, assumptions on biologic efficacy (e.g. for the second-line biologic in the treatment sequencing scenario) were required.

\section{Introduction}

Psoriatic arthritis (PsA) is a chronic, progressive, inflammatory condition that is characterised by manifestations of the joints and skin [1]. PsA can lead to irreversible joint damage, which can be highly debilitating for patients, and is associated with a considerable economic burden $[2,3]$. Conventional systemic disease-modifying anti-rheumatic drugs (csDMARDs), such as methotrexate, represent standard care for the early management of patients with active PsA, with biologics, such as tumour necrosis factor inhibitors (TNFis), providing a treatment option for those patients who have responded inadequately to prior csDMARDs $[1,4,5]$. Initiation of biologics in the treatment pathway is dependent on the number of prior csDMARDs received, amongst other factors [5, 6]. According to treatment guidelines from the British Society for Rheumatology (BSR) and the British Health Professionals in Rheumatology (BHPR) (2012), TNFi therapy is typically recommended for patients who have failed at least two csDMARDs [7]. However, the guidelines also recommend that TNFis may be considered after failure of only one csDMARD if there is evidence of adverse prognostic factors, such as the presence of five or more swollen joints in association with elevated C-reactive protein persisting for $\geq 3$ months, or structural joint damage due to disease [7]. More recent guidelines from the European League Against Rheumatism (EULAR) (2015) recommend that biologics should be considered following an inadequate response to at least one csDMARD for patients with peripheral arthritis [8].

Secukinumab is a fully human immunoglobulin $\operatorname{IgG} 1 / \kappa$ monoclonal antibody that selectively binds to and neutralises the proinflammatory cytokine interleukin (IL)-17A [9]. The efficacy and tolerability of secukinumab in adult patients with active PsA despite prior csDMARDs has been demonstrated in two phase 3 placebo-controlled trials (FUTURE 1 and FUTURE 2) $[10,11]$. In both trials, secukinumab $150 \mathrm{mg}$ met the same primary endpoint, with a higher proportion of secukinumab-treated patients achieving an American College of Rheumatology 20 response compared to the placebo group at week $24[10,11]$. Response to treatment in both trials was also evaluated using the Psoriatic Arthritis Response Criteria (PsARC), which is used to define treatment response in guidance from the National Institute for Health and Care Excellence (NICE) [5, 6, 10, 11]. The Psoriasis Area Severity Index (PASI) and Health Assessment Questionnaire (HAQ) were used to assess skin outcomes and functional ability, respectively.

The cost effectiveness of secukinumab as a treatment for patients with psoriasis in the UK has been evaluated previously and has also been demonstrated in TNFi-naïve and TNFi-experienced PsA patients in analyses conducted from the Canadian healthcare perspective $[12,13]$. Here, the cost effectiveness of secukinumab as a treatment for active PsA in adults who have responded inadequately to prior csDMARDs has been evaluated from the perspective of the UK National Health Service (NHS). As biologics may be initiated at various places in the treatment pathway, separate analyses have been conducted based on the number of prior csDMARDs to which patients have responded inadequately, in a similar manner to the UK analysis by Corbett et al. [14]. The analyses presented include hitherto unpublished utilities derived from a novel algorithm. Furthermore, an alternative approach to modelling second-line biologics has been explored in scenario analyses.

\section{Methods}

\subsection{Population, Interventions and Comparators}

The economic evaluation considered adults with active PsA who were TNFi-naive and without concomitant moderate-to-severe psoriasis, and who had responded 
inadequately to prior csDMARDs. Two patient subpopulations were considered separately in the analysis: those who had (a) failed treatment with one prior csDMARD (1 prior csDMARD) and (b) failed treatment with two or more prior csDMARDs ( $\geq 2$ prior csDMARDs). For both subpopulations, patients entered the model with an average age of 48 years and had a mean weight of $87.11 \mathrm{~kg}$ (standard deviation, 19.66); $48 \%$ of the modelled cohort were male. These demographic characteristics were based on the randomised trial population of FUTURE 2 [11].

The intervention of interest was secukinumab $150 \mathrm{mg}$, which is the licensed dose for patients without concomitant moderate-to-severe psoriasis and for those who are TNFi naïve [9]. This dose was included in FUTURE 2 and inputs regarding patient characteristics and treatment response have been derived from this trial rather than FUTURE 1, which used an unlicensed intravenous loading dose $[10,11]$.

The relevant comparators for each subpopulation were selected based on published guidance from UK Health Technology Assessment (HTA) bodies for the treatment of active PsA following an inadequate response to prior csDMARDs [4-6, 15, 16]. Current NICE and Scottish Medicines Consortium (SMC) recommendations for biologics require patients to have had an inadequate response to two or more csDMARDs; therefore the only comparator included for the 1 prior csDMARD subpopulation was standard of care (SoC) (methotrexate, $25 \mathrm{mg}$ per week, in accordance with 2015 EULAR guidelines) [4-6, 8, 15, 16]. For the $\geq 2$ prior csDMARDs subpopulation, the TNFis (adalimumab, certolizumab pegol, etanercept, infliximab, and golimumab) were included, with each TNFi modelled according to the licensed dose. The golimumab dose (50 mg or $100 \mathrm{mg}$ ) did not result in significant differences in efficacy as part of a network meta-analysis (NMA), and so the $50 \mathrm{mg}$ results were used. Ustekinumab was not included as a comparator as it is only recommended by NICE and the SMC for patients who have either had treatment with one or more TNFis already or for whom treatment with a TNFi is contraindicated [6, 17, 18]. For etanercept and infliximab, biosimilar products are available in the UK, and so both originator and biosimilar products were included as separate comparators in the analysis, with only drug acquisition costs altered for the biosimilar products. Patients receiving biologics were assumed to also receive SoC.

\subsection{Model Structure}

The model consisted of a 3-month decision tree that led into a Markov state transition model with three health states: Maintenance Treatment, SoC, and Death (see Fig. 1). This structure is consistent with previous cost effectiveness analyses of biologics for PsA, including
Rodgers et al., which has formed the basis for cost effectiveness analyses for UK HTA [4-6, 14, 19, 20].

Patients entered the decision tree on treatment initiation and response to therapy using PsARC was assessed after 3 months. The timing of the assessment was based on 2012 BSR/BHPR guideline recommendations [7]. Patients were defined as responders or non-responders depending on whether they had achieved a PsARC response at 3 months, with responders continuing treatment with biologics in the Markov model (Maintenance Treatment) and non-responders receiving SoC (as shown in Fig. 1).

During each subsequent 3-month cycle, patients in the Maintenance Treatment state could continue treatment and remain in this state, discontinue biologic therapy and thus transition to the SoC state, or die and thus enter the absorbing Death state (see Fig. 1). Withdrawal from biologic therapy was based on FUTURE 2 trial all-cause discontinuation rates for secukinumab $150 \mathrm{mg}$, with rates specified in year $1(14.0 \%)$ and all subsequent years $(11.6 \%)$. In line with the assumption made by Corbett et al., a common discontinuation rate was assumed for all biologics [14]. Patients in the SoC state were modelled to receive SoC until death and could not transition back to the Maintenance Treatment state.

The model tracked disease progression via changes over time in HAQ and PASI scores, with utility and diseaserelated costs accrued throughout the time horizon based on these scores. In utilising both HAQ and PASI scores, the model considers the impact of both arthritic and skin components of PsA on patients' health-related quality of life and healthcare resource use. Treatment effect was modelled as the change from baseline in HAQ and the distribution of PASI scores at 3 months (see Sect. 2.3), with the former conditioned on whether or not a patient had achieved a PsARC response at 3 months. In order to isolate the clinical effect of each treatment from the general benefit of trial participation, both PASI and HAQ scores were adjusted to reflect the response observed by patients receiving placebo, as per Rodgers et al. [20]. PASI and HAQ scores were assumed to remain unchanged whilst patients continued to receive biologic therapy in the Maintenance Treatment state, again consistent with Rodgers et al. [20]. Sustained improvements in HAQ have also been reported in FUTURE 2 [11]. Patients who withdrew from biologic therapy and entered the SoC state were modelled to rebound to baseline PASI and HAQ scores in the cycle after treatment withdrawal (i.e. rebound was set equal to initial gain in the base case analysis). In each subsequent cycle, these patients were modelled to experience a steady, linear increase in their HAQ score $(0.018$ per cycle) until death or the maximum HAQ score of three was reached, to reflect worsening arthritis whilst not receiving biologics $[19,20]$. 
Fig. 1 Model structure. a Decision tree model structure. Responders were those patients who achieved a PsARC response at 3 months. b Markov model structure. PsARC psoriatic arthritis response criteria, $S o C$ standard of care

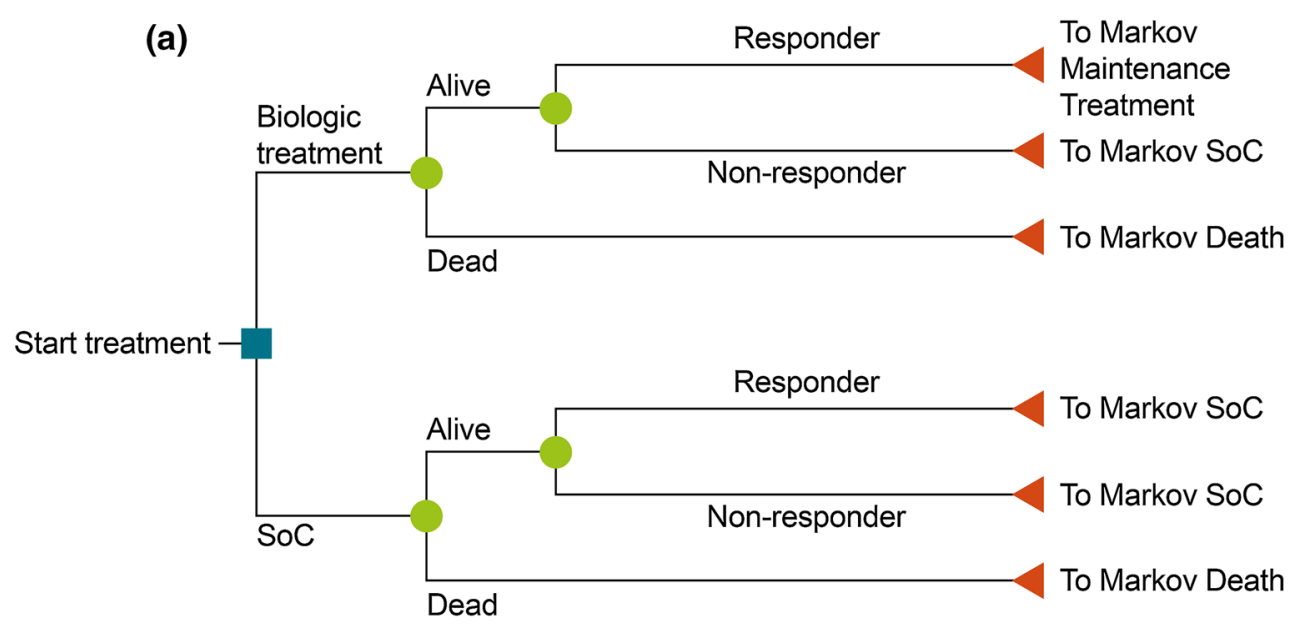

(b)

The model was conducted over a 40-year time horizon from the perspective of the UK NHS. This time horizon was chosen to reflect the chronic nature of the condition. Costs and utilities were accrued across the time horizon and were assigned to health states either directly (e.g. cost associated with biologics or SoC) or via other factors related to treatment, such as disease scores (HAQ and PASI).

\subsection{Modelling of Health Outcomes}

\subsubsection{Baseline Clinical Characteristics}

For both subpopulations included in the economic evaluation, baseline HAQ (1.209) and PASI (7.304) scores were based on those of randomised patients in the FUTURE 2 trial who had not previously received TNFi therapy.

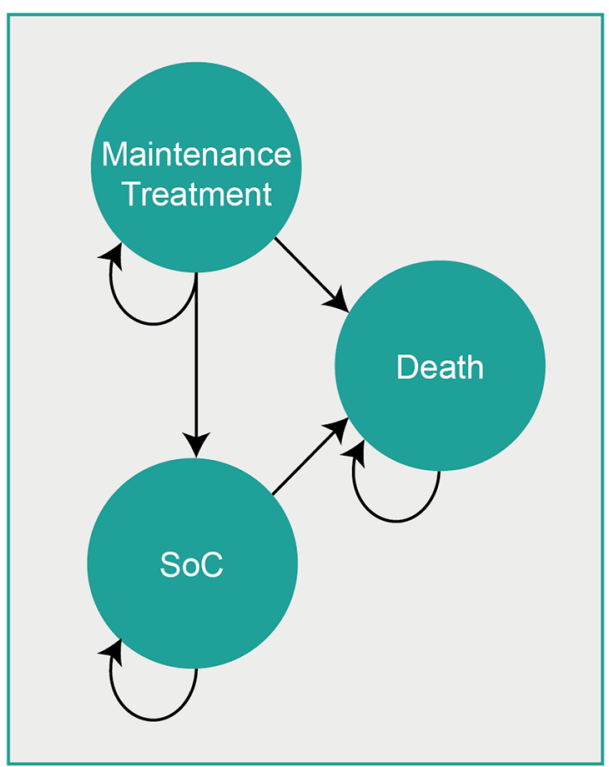

\subsubsection{PsARC Responses and PASI Distribution at 3 Months}

For secukinumab and SoC in the 1 prior csDMARD subpopulation, PsARC response and the distribution of patients with PASI response $<50$, 50-74, 75-89 and 90-99 at 3 months were based on post hoc subgroup analyses from the randomised, placebo-controlled FUTURE 2 trial (see Table 4a in the Electronic Supplementary Material). For the $\geq 2$ prior csDMARDs subpopulation, response to treatment at the end of the initial 3-month period (PsARC and PASI) was based on the results of a systematic literature review (SLR) and NMA of clinical evidence for licensed doses of biologics for PsA (see Table 5, Electronic Supplementary Material). A similar NMA is reported by McInnes et al. [21]. Differences across each PASI response category were statistically significant for secukinumab $150 \mathrm{mg}$ versus certolizumab 
pegol and etanercept, but no significant differences for secukinumab versus comparators were reported for PsARC.

In modelling the change from baseline in PASI based on response category, it was assumed that patients would achieve the minimal reduction for each category (i.e. PASI 75 responders would achieve at least a $75 \%$ reduction in baseline PASI), as per Rodgers et al. [20].

\subsubsection{Changes in $H A Q$ at 3 Months}

The extent to which HAQ scores were reduced in the model was conditional on whether or not a PsARC response had been achieved at 3 months. For secukinumab, the reduction in HAQ score on treatment response for the 1 prior csDMARD and $\geq 2$ prior csDMARDs subpopulations were based on corresponding post hoc subgroup analyses of FUTURE 2 (see Table 4b; Electronic Supplementary Material). A response-dependent change in HAQ score for SoC is also modelled based on that observed in the FUTURE 2 subgroup analyses. For TNFis, changes in HAQ were derived from another published cost effectiveness analysis [19]. As certolizumab pegol was not included in this previous cost effectiveness analysis, the average change in HAQ for all other TNFis was used in the absence of any published data.

\subsubsection{Adverse Events}

Serious infection was the only adverse event included in the model, with the probability of an event in each 3-month cycle based on trial data for each intervention (see Table 4c; Electronic Supplementary Material) [11, 22-26]. This adverse event was selected due to the increased risk of infection with biologics targeting immune pathways $[9,27]$. Five percent of serious infections were assumed to be cases of tuberculosis, which were modelled to have higher costs per event than other serious infections [28].

\subsubsection{Mortality}

Baseline mortality in the model was determined using mortality rates from the general population life tables [29]. This baseline rate was then adjusted for the gender-specific relative risk of death for patients with PsA (male 1.65 and female 1.59), as done in previous cost effectiveness analyses [20, 30].

\subsection{Utility Inputs}

Utility values for the model were derived from a linear mixed model that used patient-level EuroQol 5-Dimensions (3-Level; EQ-5D-3L) data from FUTURE 2 and estimated utility based on absolute HAQ and PASI scores, baseline EQ-5D, age, gender and prior TNFi status (see Table 6; Electronic Supplementary Material).

This approach reflects the clinical experience of patients, with lower HAQ and PASI scores being associated with higher utility values. No disutility was modelled for the occurrence of adverse events, as per the assumption made by Rodgers et al. [20].

\subsection{Cost and Resource Use Inputs}

The evaluation was conducted from the perspective of the UK NHS and so only direct costs incurred by the NHS were included in the analysis. These included drug acquisition costs (list price for all interventions), administration and monitoring costs, disease-related costs and costs associated with the management of serious infections (see Table 7; Electronic Supplementary Material). The cost year was 2017 and, where required, costs were inflated using the Hospital and Community Health Services Index [31].

\subsection{Model Outcomes}

The model outcomes included the total cost and qualityadjusted life years (QALYs) accrued over the model time horizon, with both costs and QALYs discounted at a 3.5\% annual rate, as is standard practice for UK cost effectiveness analyses [32]. A half-cycle correction using the life table method was applied to all outcomes, with the exception that drug costs and QALYs were not half-cycle corrected in the first cycle of the Markov model, as the treatment responses and resulting utility changes were achieved within 3 months. Cost effectiveness was assessed in terms of the incremental cost effectiveness ratio (ICER), which describes the incremental costs per QALY gained for secukinumab versus each comparator. A fully incremental analysis has also been conducted for the $\geq 2$ prior csDMARDs subpopulation.

\subsection{Sensitivity and Scenario Analyses}

One-way deterministic sensitivity analyses were conducted for each subpopulation to identify parameters which had the greatest influence on cost effectiveness results. Given secukinumab was generally either dominant or associated with a 'south-west' ICER, changes in cost effectiveness were assessed in terms of net monetary benefit, with one QALY valued at $£ 30,000$ to represent the upper limit of cost effectiveness that may be considered by UK decision makers. Probabilistic sensitivity analyses were also conducted in which all model parameters were varied simultaneously over 1000 simulations. Inputs for these 
sensitivity analyses are presented in Table 8 (see the Electronic Supplementary Material).

To test alternative assumptions, various scenarios were conducted including: varying the time horizon used; including PASI in the criteria for treatment response; removing the placebo adjustment effect on HAQ and PASI; assuming that HAQ rebound is equal to natural history at the time of discontinuation; and using utility values from Rodgers et al. [20]. Additional scenarios were also conducted in the $\geq 2$ prior csDMARDs subpopulation only, including: the use of a second-line biologic on discontinuation, as recommended in the 2012 BSR/BHPR guidelines; the inclusion of publicly-available Patient Access Schemes (PASs) for certolizumab pegol and golimumab (no PASs exist for the other biologics, with the exception of a confidential PAS for secukinumab); and assuming equivalence with secukinumab for any non-significant differences in PASI and PsARC [4, 7, 15]. As part of the treatment sequencing scenarios, the second-line biologic was modelled as a weighted average of all biologics (other than that received first-line) based on estimated use of each biologic in clinical practice. Scenarios were conducted in which (a) biologics were assumed to have equivalent efficacy in first- and second-line, and (b) a $20 \%$ reduction in each efficacy input was modelled in the second line.

\subsection{Model Validation}

Quality control for model internal validity was performed through independent review of the model by a different health economist to those responsible for initial construction of the model. A rheumatologist (B. K.) specialising in PsA was also involved in the development of the model to ensure that the underlying assumptions and model outcomes were reflective of UK clinical practice.

\section{Results}

\subsection{Base Case Results}

In the 1 prior csDMARD subpopulation, secukinumab was associated with a QALY gain of 1.030 versus SoC, but was also associated with higher total costs over the 40-year model time horizon (incremental cost of $£ 29,619$; see Table 1a). The ICER for secukinumab at list price versus SoC in the 1 prior csDMARD subpopulation was $£ 28,748$ per QALY gained.

In the $\geq 2$ prior csDMARDs subpopulation, secukinumab dominated certolizumab pegol, etanercept and golimumab (see Table $1 \mathrm{~b}$ for pairwise comparisons and fully incremental results). Secukinumab was also associ- ated with lower total costs versus infliximab, but produced marginally fewer QALYs (0.026) over the model time horizon. The ICERs for secukinumab versus infliximab corresponded to savings in excess of $£ 1$ million per QALY foregone with secukinumab, with the ICER being positioned in the south-west quadrant of the cost effectiveness plane. Compared to adalimumab, secukinumab was associated with higher total costs and a greater number of QALYs over the model time horizon, which resulted in an ICER of $£ 5680$ per QALY gained for secukinumab versus adalimumab. The use of biosimilar prices for etanercept and infliximab did not meaningfully alter the results for secukinumab versus these TNFis. The fully incremental analysis found all interventions other than secukinumab, adalimumab (the least costly intervention) and infliximab (biosimilar) to be dominated or extendedly dominated. The ICER for secukinumab versus adalimumab was $£ 5680$ per QALY gained, and the ICER for infliximab (biosimilar) versus secukinumab was $£ 1,147,641$ per QALY gained.

In both populations, differences in total cost were largely due to biologic costs (see Table 9; Electronic Supplementary Material).

\subsection{Sensitivity and Scenario Analyses}

In both subpopulations, the model parameters associated with HAQ changes were generally the most influential factors on cost effectiveness results. Tornado plots for secukinumab versus comparators in the 1 prior csDMARD and $\geq 2$ prior csDMARDs subpopulations are presented in Fig. 2 and Fig. 5 (Electronic Supplementary Material), respectively. The $95 \%$ confidence intervals for incremental results from the probabilistic analysis are provided in Table 2 and suggest that the model results are generally robust to combined uncertainty in parameter values. Figure 3 shows the scatter plot of all probabilistic results for secukinumab versus SoC in the 1 prior csDMARD subpopulation (for the $\geq 2$ prior csDMARDs subpopulation, see Fig. 6 in the Electronic Supplementary Material). The probability of secukinumab having the highest net monetary benefit compared to all comparators was $48.9 \%$ and $88.9 \%$ in the 1 prior csDMARD and $\geq 2$ prior csDMARDs subpopulations, respectively, when one QALY was valued at between $£ 20,000$ and $£ 30,000$. Cost effectiveness acceptability curves are presented in Fig. 4 and Fig. 7 (Electronic Supplementary Material) for the 1 prior csDMARD and $\geq 2$ prior csDMARDs subpopulations, respectively.

The ICERs for secukinumab versus comparators in each of these scenario analyses are presented in Table 3 ( 1 prior csDMARD) and Table 10 ( $\geq 2$ prior csDMARDs; Electronic Supplementary Material). In the majority of sce- 
Table 1 Base case deterministic cost effectiveness results (with incremental results for secukinumab vs each comparator and results of fully incremental analyses)

\begin{tabular}{|c|c|c|c|c|c|c|}
\hline \multirow{2}{*}{$\begin{array}{l}\text { Intervention } \\
\text { SEC }\end{array}$} & Total costs $(\mathfrak{£})$ & \multicolumn{2}{|l|}{ Total QALYs } & Incremental costs $(\mathfrak{E})$ & \multirow[t]{2}{*}{ Incremental QALYs } & \multirow[t]{2}{*}{ ICER ( $£$ per QALY) } \\
\hline & 91,062 & 7.837 & & & & \\
\hline SoC & 61,443 & 6.807 & & & 1.030 & 28,748 \\
\hline \multicolumn{7}{|c|}{ b. $\geq 2$ prior csDMARDs } \\
\hline Intervention & $\begin{array}{l}\text { Total } \\
\text { costs }(£)\end{array}$ & $\begin{array}{l}\text { Total } \\
\text { QALYs }\end{array}$ & $\begin{array}{l}\text { Incremental } \\
\text { costs }(£)\end{array}$ & $\begin{array}{l}\text { Incremental } \\
\text { QALYs }\end{array}$ & $\begin{array}{l}\text { ICER ( } £ \text { per QALY), } \\
\text { pairwise }\end{array}$ & $\begin{array}{l}\text { ICER ( } £ \text { per QALY), } \\
\text { fully incremental }\end{array}$ \\
\hline SEC & 93,113 & 7.998 & & & & 5680 \\
\hline ADA & 90,993 & 7.615 & 2120 & 0.373 & 5680 & - \\
\hline $\mathrm{CZP}$ & 101,246 & 7.913 & -8134 & 0.074 & Dominated by SEC & Dominated \\
\hline ETN (originator) & 100,134 & 7.984 & -7022 & 0.004 & Dominated by SEC & Dominated \\
\hline ETN (biosimilar) & 96,509 & 7.984 & -3396 & 0.004 & Dominated by SEC & Dominated \\
\hline GOL & 104,772 & 7.709 & $-11,659$ & 0.279 & Dominated by SEC & Dominated \\
\hline INF (originator) & 128,949 & 8.013 & $-35,837$ & -0.026 & $1,403,849^{\mathrm{a}}$ & Extendedly dominated \\
\hline INF (biosimilar) & 122,409 & 8.013 & $-29,296$ & -0.026 & $1,147,641^{\mathrm{a}}$ & $1,147,641$ \\
\hline
\end{tabular}

Incremental results are presented for SEC vs comparator(s). In the $\geq 2$ prior csDMARD subpopulation, the fully incremental analysis found all interventions other than SEC, ADA (least costly) and INF (biosimilar) to be dominated or extendedly dominated (INF [originator]). The ICER for SEC vs ADA was $£ 5680$ per QALY gained, and the ICER for INF (biosimilar) vs SEC was $£ 1,147,641$ per QALY gained. INF (originator) was extendedly dominated by SEC and INF (biosimilar)

$A D A$ adalimumab, $c S D M A R D$ conventional systemic disease-modifying anti-rheumatic drug, $C Z P$ certolizumab pegol, ETN etanercept, $G O L$ golimumab, ICER incremental cost effectiveness ratio, INF infliximab, $Q A L Y$ quality-adjusted life year, SEC secukinumab, SoC standard of care

${ }^{a}$ The ICERs for SEC vs INF (originator and biosimilar) land in the south-west quadrant of the cost effectiveness plane (negative incremental costs and negative incremental QALYs). Therefore, the ICER is a positive value and should be interpreted as the cost savings per QALY foregone with SEC vs INF

narios explored, secukinumab was associated with an ICER $<£ 30,000$ per QALY gained (or an ICER in the south-west quadrant $>£ 30,000$ per QALY gained) versus each comparator and secukinumab at list price was still dominant compared to certolizumab pegol and golimumab when the PASs for these comparators were included in the $\geq 2$ prior csDMARDs subpopulation. Results varied when considering different response definitions (i.e. PsARC and PASI) and on modelling of a second-line biologic. Reducing the time horizon to 10 years and assuming that HAQ rebound was equal to natural history resulted in considerably higher ICERs than in the base case analysis in the 1 prior csDMARD subpopulation.

\section{Discussion}

Based on the deterministic results of the analysis, decision makers may consider secukinumab $150 \mathrm{mg}$ to represent a cost-effective use of UK NHS resources for patients with active PsA who are TNFi-naive and without concomitant moderate-to-severe psoriasis, and who have responded inadequately to prior csDMARDs. The inclusion of separate analyses for patients according to the number of prior csDMARDs has allowed cost effectiveness to be evaluated at specific stages of the treatment pathway.

Results from the 1 prior csDMARD subpopulation, in which secukinumab was shown to have an ICER of less than $£ 30,000$ per QALY gained versus SoC, should be of interest to clinicians and payers in the UK given the recent clinical guideline recommendations for the use of biologics after an inadequate response to one prior csDMARD [7, 8]. In the $\geq 2$ prior csDMARDs subpopulation, secukinumab was shown to dominate biologic comparators, including golimumab, certolizumab pegol and etanercept. The cost effectiveness of secukinumab versus infliximab and etanercept was also demonstrated when the prices for these comparators were based on biosimilar products. The availability of a confidential PAS discount for secukinumab in the UK further strengthens the case for cost effectiveness in both subpopulations [15]. In conducting the analysis from a UK perspective and using UK 
HAQ change after long-term biologic withdrawa

FUTURE 2 HAQ disability index score

HAQ change W/ PSARC response - Placebo Cost per change in $\mathrm{HAQ}$

HAQ change W/ PSARC response - SEC 150

3-month PSARC response - SoC 3-month PSARC response - SEC 150 FUTURE 2 coefficient PASI total score PsA mortality relative risk - males PSA mortality relative risk - females Outpatient rheumatology cost Yr 1 discontinuation rate - SEC 150

Yr 2 discontinuation rate - SEC 150 Cost for uncontrolled psoriasis Infection probability - SEC 150

\section{$\square$ Lower bound of input parameter \\ Higher bound of input parameter}

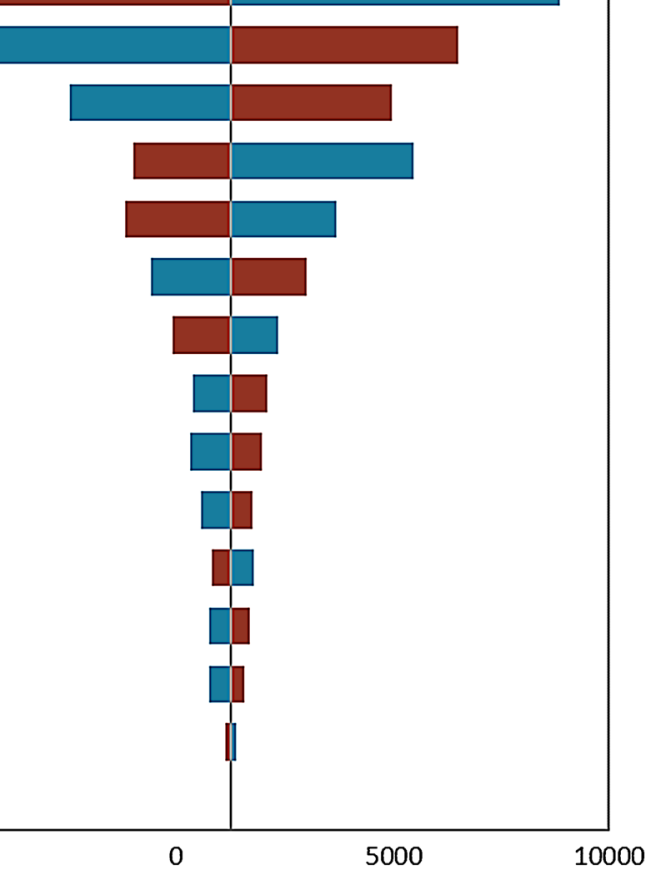

Net Monetary Benefit

Fig. 2 One-way sensitivity analysis in the 1 prior csDMARD subpopulation: tornado plot for secukinumab vs SoC. A single QALY was valued at $£ 30,000$. csDMARD conventional systemic disease-modifying anti-rheumatic drug, HAQ Health Assessment
Questionnaire, PASI Psoriasis Area Severity Index, PsA psoriatic arthritis, $P s A R C$ Psoriatic Arthritis Response Criteria, $Q A L Y$ qualityadjusted life year, $S E C$ secukinumab, $S o C$ standard of care, $Y r$ year

Table 2 Probabilistic cost effectiveness results (with incremental results for secukinumab vs each comparator) [average (95\% confidence interval)]

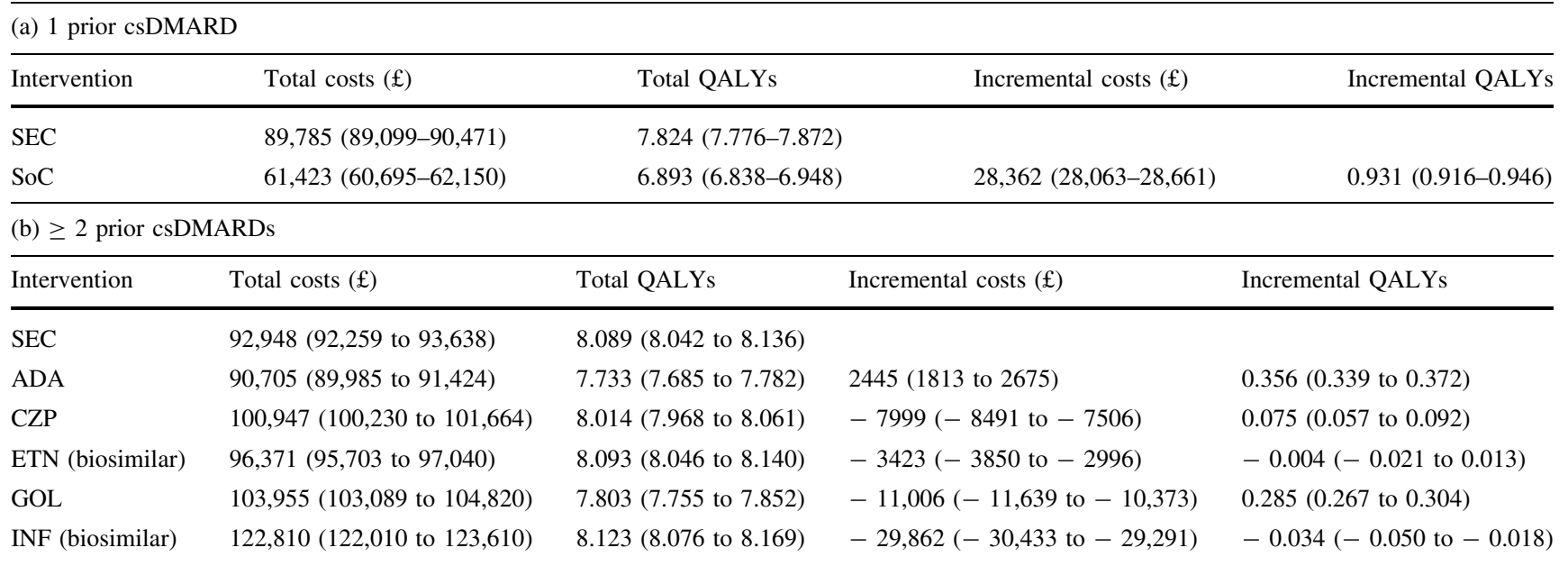

Incremental results are presented for SEC vs comparator(s)

$A D A$ adalimumab, $C Z P$ certolizumab pegol, $c S D M A R D$ conventional systemic disease-modifying anti-rheumatic drug, ETN etanercept, $G O L$ golimumab, $I N F$ infliximab, $Q A L Y$ quality-adjusted life year, SEC secukinumab, $S o C$ standard of care

guidelines and NICE recommendations to inform the model, the analyses are most relevant to the UK NHS. Analyses using other country perspectives (e.g. Canada) have been published elsewhere [13].
The results of the cost effectiveness analysis were seen to be generally robust to changes in model parameters and assumptions, as demonstrated in probabilistic sensitivity analyses and a number of scenario analyses. In cases where 


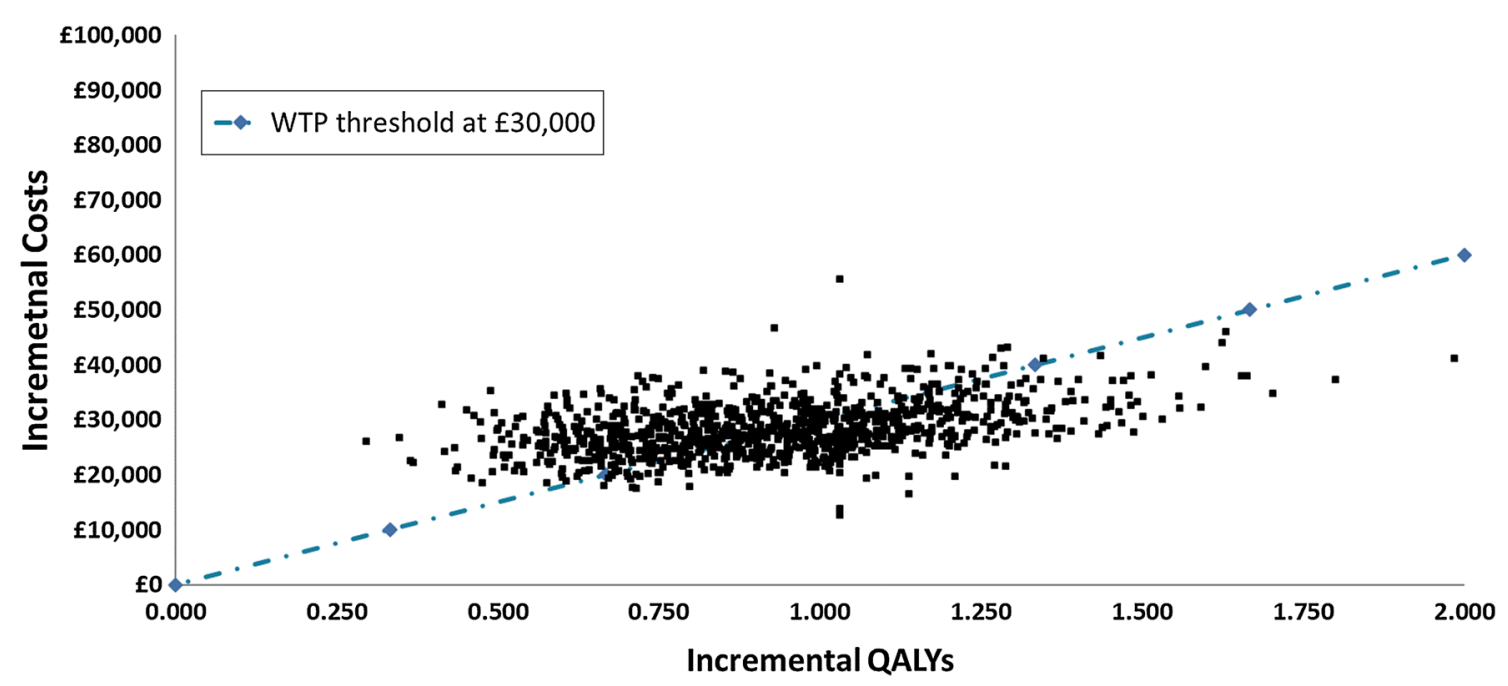

Fig. 3 Probabilistic results in the 1 prior csDMARD subpopulation: scatter plot for secukinumab vs SoC. $c s D M A R D$ conventional systemic disease-modifying anti-rheumatic drug, $Q A L Y$ quality-adjusted life year, $S o C$ standard of care, WTP willingness to pay

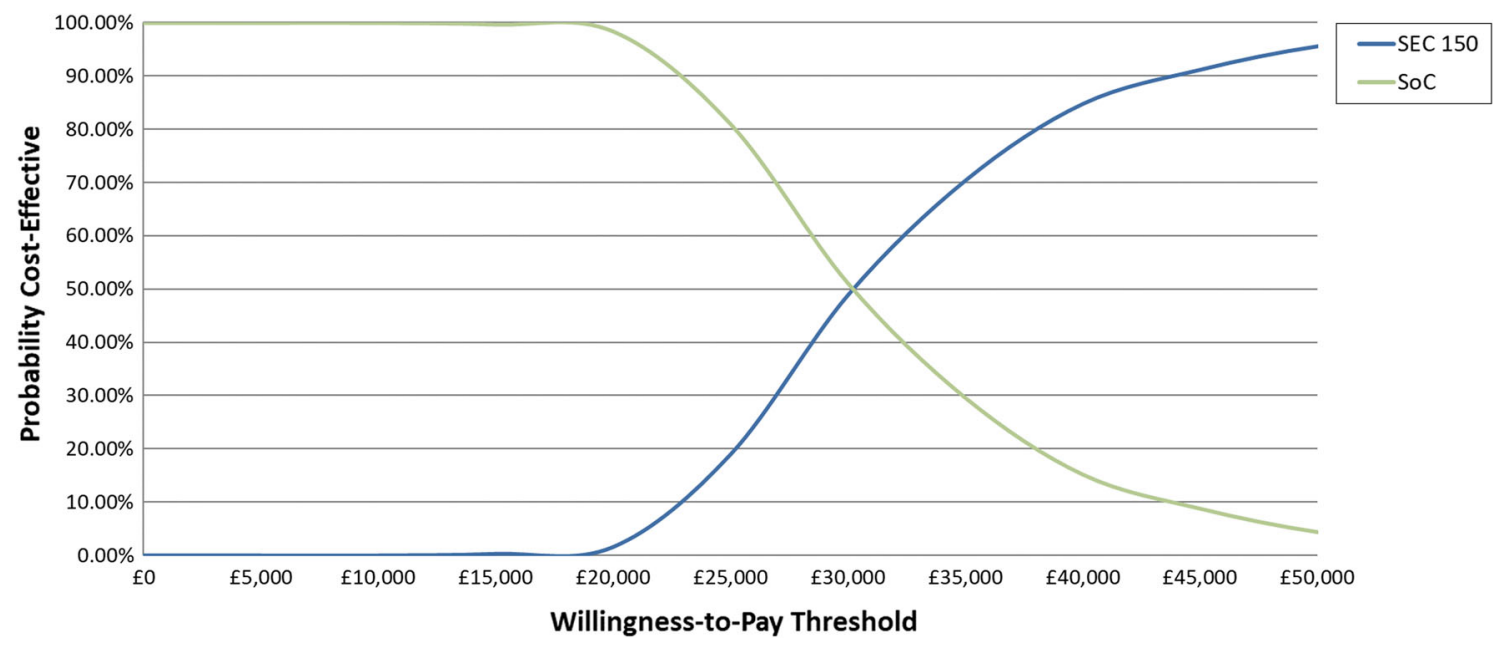

Fig. 4 Probabilistic results in the 1 prior csDMARD subpopulation: cost effectiveness acceptability curve for secukinumab vs SoC. $c s D M A R D$ conventional systemic disease-modifying anti-rheumatic drug, SEC secukinumab, SoC standard of care

cost effectiveness results were seen to differ considerably between base case and scenario analyses (e.g. time horizon and HAQ rebound assumptions in the 1 prior csDMARD subpopulation and response definition in the $\geq 2$ prior csDMARDs subpopulation), the choice of base case assumptions was consistent with previous economic evaluations $[14,20]$. As shown in probabilistic analyses, uncertainty in the cost effectiveness results was higher in the 1 prior csDMARD subpopulation.

The decision model is based on a well-established model structure that characterises the impact of biologics on both the skin and arthritic components of PsA. Another strength of the model is the use of clinical evidence derived from patient-level data for secukinumab and an SLR for comparators. Despite the comprehensiveness of the review, a number of assumptions were required for some clinical parameters due to limited published data for comparators. The inclusion of TNFi-experienced patients was a major source of between-trial variation and it is likely that treatment response in the overall population of more recent trials (such as FUTURE 2), which included TNFi-experienced patients, would be affected by the inclusion of these patients [11]. Sequencing scenario analyses were also restricted by the lack of published data for comparator biologics in TNFi-experienced patients, hence the modelling of only first-line biologics in the base case analysis.

The results from the 1 prior csDMARD analysis are consistent with those from the UK cost effectiveness analysis presented by Corbett et al., in which secukinumab $150 \mathrm{mg}$ was reported to have ICERs versus SoC of less than $£ 30,000$ per QALY in the relevant 1 prior csDMARD 
Table 3 Scenario analyses: ICERs for the 1 prior csDMARD population (secukinumab vs SoC)

\begin{tabular}{llll}
\hline$\#$ & Variable/assumption explored (base case) & Scenario & ICER (£ per QALY) \\
\hline \multicolumn{2}{l}{ Base case } & & 28,748 \\
$1 \mathrm{a}$ & Time horizon (40 years) & 10 years & 56,705 \\
$1 \mathrm{~b}$ & & 20 years & 36,272 \\
$1 \mathrm{c}$ & & 52 years & 28,701 \\
$2 \mathrm{a}$ & Response definition (PsARC only) & PsARC and PASI $\geq 50$ & 26,658 \\
$2 \mathrm{~b}$ & & PsARC and PASI $\geq 75$ & 27,654 \\
$2 \mathrm{c}$ & & PsARC and PASI $\geq 90$ & 31,226 \\
3 & Adjust HAQ for placebo (yes) & No & 25,110 \\
4 & Adjust PASI for placebo (yes) & No & 28,680 \\
5 & HAQ rebound (equal to initial gain) & HAQ rebound is equal to natural history at the time of discontinuation & 49,149 \\
6 & Utility source (FUTURE 2) & Rodgers et al. & 16,522 \\
\hline
\end{tabular}

csDMARD conventional systemic disease-modifying anti-rheumatic drugs, $H A Q$ Health Assessment Questionnaire, ICER incremental cost effectiveness ratio, PASI Psoriasis Area Severity Index, PsARC Psoriatic Arthritis Response Criteria, SoC standard of care

${ }^{a} \mathrm{HAQ}$ rebounds to the level and subsequent trajectory it would have been if there had been no initial response, as described by Rodgers et al. [20]

subpopulations (no psoriasis or mild to moderate psoriasis in Corbett et al. [14]). Although secukinumab $150 \mathrm{mg}$ was associated with lower costs versus other biologics in the relevant $\geq 2$ prior csDMARDs subpopulations in both Corbett et al. and here, in Corbett et al., secukinumab was associated with fewer QALYs versus other biologics [14]. Differences in total QALYs in the $\geq 2$ prior csDMARDs subpopulation are likely to reflect differences in the NMAs utilised in each model [14]. Other differences that may have affected results include the use of different utilities; the consideration of individual psoriasis severity subgroups by Corbett et al.; and the use of treatment sequencing in the base case analysis of Corbett et al. [14]. Although using an established model framework, this economic evaluation makes use of alternative sources of inputs, including a novel utility algorithm which included baseline EQ-5D, age, gender and TNFi status as coefficients, and explores a simple approach to treatment sequencing in which the relative use of alternative biologics, with either unchanged or waned efficacy in the second-line setting, is considered. Results from these scenarios differed to the base case, with secukinumab dominant versus adalimumab but no longer dominant versus etanercept, thus demonstrating the impact of treatment sequencing on cost effectiveness results. Neither this model nor Corbett et al. incorporated the longterm benefits that biologics, such as secukinumab, may have in delaying or preventing the occurrence of structural joint damage associated with PsA [14, 33]. Furthermore, assumptions were required in both models regarding changes over time in PASI and HAQ, raising a potential need for further consideration of how the long-term effects of biologics can be most appropriately captured in cost effectiveness models.

\section{Conclusion}

Based on the evaluation here, secukinumab $150 \mathrm{mg}$ at list price may be considered cost effective for PsA patients in the UK who are TNFi-naive and without concomitant moderate-to-severe psoriasis, and who have responded inadequately to either one or two or more prior csDMARDs. The availability of data for all biologics at each stage of the treatment pathway would reduce uncertainties in the cost effectiveness analyses of biologics after prior csDMARDs.

Data availability statement The datasets used for the cost effectiveness analysis are not publicly available, but may be available from the corresponding author on reasonable request.

Acknowledgements The authors acknowledge Costello Medical, $\mathrm{UK}$, for writing and editorial assistance.

Author contributions CG and LM were involved in model conceptualisation, design, development and validation and provided technical input on the publication content. VB and WS were involved in development and validation of the model and provided technical input on the publication content. SMJ and PG were involved in the model conceptualisation and provided input on the publication content. $\mathrm{AH}$ was involved in model validation and was responsible for defining and developing the publication content. BK provided clinical input on model design and validation, and on the publication content.

\section{Compliance with Ethical Standards}

Conflict of interest VB, WS, CG and LM declare they have no conflicts of interest. VB was (at the time of the study) and WS is an employee of BresMed Health Solutions, and CG and LM are employees of RTI Health Solutions; these organisations were contracted by Novartis Pharmaceuticals UK Ltd to undertake part of the work. SMJ is an employee of Novartis Pharma AG, Basel, Switzerland, and owns stock and stock options in Novartis Pharma AG, 
Basel, Switzerland. PG is an employee of Novartis Healthcare Private Limited, Hyderabad, India. AH is an employee of Novartis Pharmaceuticals UK Limited, Camberley, UK. BK has received research support from Abbvie, Eli Lilly \& Co, Novartis, Roche, and UCB; honoraria from Eli Lilly \& Co, Novartis, and Sandoz; and speaker fees from BMS, Janssen, Novartis, and Pfizer.

Funding This study was funded by Novartis Pharmaceuticals UK Ltd, Camberley, UK.

Open Access This article is distributed under the terms of the Creative Commons Attribution-NonCommercial 4.0 International License (http://creativecommons.org/licenses/by-nc/4.0/), which permits any noncommercial use, distribution, and reproduction in any medium, provided you give appropriate credit to the original author(s) and the source, provide a link to the Creative Commons license, and indicate if changes were made.

\section{References}

1. Mease PJ, Armstrong AW. Managing patients with psoriatic disease: the diagnosis and pharmacologic treatment of psoriatic arthritis in patients with psoriasis. Drugs. 2014;74(4):423-41. https://doi.org/10.1007/s40265-014-0191-y.

2. Gladman DD, Antoni C, Mease P, Clegg DO, Nash P. Psoriatic arthritis: epidemiology, clinical features, course, and outcome. Annals of the rheumatic diseases. 2005;64(Suppl 2):ii14-7. https://doi.org/10.1136/ard.2004.032482.

3. D'Angiolella LS, Cortesi PA, Lafranconi A, Micale M, Mangano S, Cesana G, et al. Cost and cost effectiveness of treatments for psoriatic arthritis: a systematic literature review. Pharmacoeconomics. 2018;36(5):567-89. https://doi.org/10.1007/s40273-0180618-5.

4. National Institute for Health and Care Excellence (NICE). TA220: Golimumab for the treatment of psoriatic arthritis (2011). https://www.nice.org.uk/guidance/ta220. Accessed 16 May 2017.

5. National Institute for Health and Care Excellence (NICE). TA199: Etanercept, infliximab and adalimumab for the treatment of psoriatic arthritis (2010). https://www.nice.org.uk/guidance/ ta199. Accessed 16 May 2017.

6. National Institute for Health and Care Excellence (NICE). TA340: Ustekinumab for treating active psoriatic arthritis (2015). https://www.nice.org.uk/guidance/ta340. Accessed 16 May 2017.

7. Coates LC, Tillett W, Chandler D, Helliwell PS, Korendowych E, Kyle S, et al. The 2012 BSR and BHPR guideline for the treatment of psoriatic arthritis with biologics. Rheumatology (Oxford, England). 2013;52(10):1754-7. https://doi.org/10.1093/ rheumatology/ket187.

8. Gossec L, Smolen JS, Ramiro S, de Wit M, Cutolo M, Dougados $\mathrm{M}$, et al. European League Against Rheumatism (EULAR) recommendations for the management of psoriatic arthritis with pharmacological therapies: 2015 update. Ann Rheum Dis. 2016;75(3):499-510. https://doi.org/10.1136/annrheumdis-2015208337.

9. European Medicines Agency (EMA). Cosentyx: EPAR—Product Information. http://www.ema.europa.eu/ema/index.jsp?curl= pages/medicines/human/medicines/003729/human_med_001832. jsp\&mid=WC0b01ac058001d124. Accessed 16 May 2017.

10. Mease PJ, McInnes IB, Kirkham B, Kavanaugh A, Rahman P, van der Heijde D, et al. Secukinumab inhibition of interleukin17A in patients with psoriatic arthritis. $N$ Engl J Med. 2015;373(14):1329-39. https://doi.org/10.1056/
11. McInnes IB, Mease PJ, Kirkham B, Kavanaugh A, Ritchlin CT, Rahman P, et al. Secukinumab, a human anti-interleukin-17A monoclonal antibody, in patients with psoriatic arthritis (FUTURE 2): a randomised, double-blind, placebo-controlled, phase 3 trial. Lancet (London, England). 2015;386(9999):1137-46. https://doi.org/10.1016/s01406736(15)61134-5.

12. National Institute for Health and Care Excellence (NICE). TA350: Secukinumab for treating moderate to severe plaque psoriasis (2015). https://www.nice.org.uk/guidance/ta350. Accessed 16 May 2017.

13. Goeree R, Chiva-Razavi S, Gunda P, Graham CN, Miles L, Nikoglou E, et al. Cost-effectiveness analysis of secukinumab for the treatment of active psoriatic arthritis: a Canadian perspective. J Med Econ. 2017. https://doi.org/10.1080/13696998.2017. 1384737.

14. Corbett $\mathrm{M}$, et al. Certolizumab pegol and secukinumab for treating active psoriatic arthritis following inadequate response to disease-modifying antirheumatic drugs: a systematic review and economic evaluation. Health Technol Assess. 2017;21(56):1-326.

15. National Institute for Health and Care Excellence (NICE). TA445: Certolizumab pegol and secukinumab for treating active psoriatic arthritis after inadequate response to DMARDs (2017). https://www.nice.org.uk/guidance/ta445. Accessed 24 May 2017.

16. Scottish Medicines Consortium. Advice ID 674/11: golimumab (Simponi). Treatment of active and progressive psoriatic arthritis in adult patients. https://www.scottishmedicines.org.uk/SMC Advice/Advice/674_11_golimumab_Simponi/golimumab simponi_Re_Submission. Accessed 25 Aug 2017.

17. Scottish Medicines Consortium. Advice ID 944/14: ustekinumab (Stelara). Alone or in combination with methotrexate, for the treatment of active psoriatic arthritis in adult patients when the response to previous non-biological disease-modifying antirheumatic drug therapy has been inadequate. https://www. scottishmedicines.org.uk/SMC_Advice/Advice/944_14_ ustekinumab_Stelara/ustekinumab_Stelara. Accessed 25 Aug 2017.

18. O'Connor J, Rice S, Smith A, Rodgers M, Lopez RR, Craig D, et al. The clinical and cost effectiveness of ustekinumab for the treatment of psoriatic arthritis: a critique of the evidence. Pharmacoeconomics. 2016;34(4):337-48. https://doi.org/10.1007/ s40273-015-0350-3.

19. Cawson MR, Mitchell SA, Knight C, Wildey H, Spurden D, Bird $\mathrm{A}$, et al. Systematic review, network meta-analysis and economic evaluation of biological therapy for the management of active psoriatic arthritis. BMC Musculoskelet Disord. 2014;15:26. https://doi.org/10.1186/1471-2474-15-26.

20. Rodgers M, Epstein D, Bojke L, Yang H, Craig D, Fonseca T, et al. Etanercept, infliximab and adalimumab for the treatment of psoriatic arthritis: a systematic review and economic evaluation. Health Technol Assess (Winchester, England). 2011;15(10):ixxi. https://doi.org/10.3310/hta15100 (1-329).

21. McInnes IB, Nash P, Ritchlin C, Thom H, Kanters S, Palaka E, et al. THU0437 Secukinumab for the treatment of psoriatic arthritis: comparative effectiveness results versus licensed biologics and apremilast from a network meta-analysis. Ann Rheum Dis. 2016;75(Suppl 2):348-9. https://doi.org/10.1136/ annrheumdis-2016-eular.1716.

22. Mease PJ, Fleischmann R, Deodhar AA, Wollenhaupt J, Khraishi M, Kielar D, et al. Effect of certolizumab pegol on signs and symptoms in patients with psoriatic arthritis: 24-week results of a phase 3 double-blind randomised placebo-controlled study (RAPID-PsA). Ann Rheum Dis. 2014;73(1):48-55. https://doi. org/10.1136/annrheumdis-2013-203696. NEJMoa1412679. 
23. Sterry W, Ortonne JP, Kirkham B, Brocq O, Robertson D, Pedersen $\mathrm{RD}$, et al. Comparison of two etanercept regimens for treatment of psoriasis and psoriatic arthritis: PRESTA randomised double blind multicentre trial. BMJ (Clin Res Ed). 2010;340:c147. https://doi.org/10.1136/bmj.c147.

24. Burmester GR, Panaccione R, Gordon KB, McIlraith MJ, Lacerda AP. Adalimumab: long-term safety in 23458 patients from global clinical trials in rheumatoid arthritis, juvenile idiopathic arthritis, ankylosing spondylitis, psoriatic arthritis, psoriasis and Crohn's disease. Ann Rheum Dis. 2013;72(4):517-24. https://doi. org/10.1136/annrheumdis-2011-201244.

25. Torii $H$, Nakagawa $H$. Infliximab monotherapy in Japanese patients with moderate-to-severe plaque psoriasis and psoriatic arthritis. A randomized, double-blind, placebo-controlled multicenter trial. J Dermatol Sci. 2010;59(1):40-9. https://doi.org/10. 1016/j.jdermsci.2010.04.014.

26. Kavanaugh A, van der Heijde D, McInnes IB, Mease P, Krueger GG, Gladman DD, et al. Golimumab in psoriatic arthritis: oneyear clinical efficacy, radiographic, and safety results from a phase III, randomized, placebo-controlled trial. Arthritis Rheum. 2012;64(8):2504-17. https://doi.org/10.1002/art.34436.

27. Galloway JB, Hyrich KL, Mercer LK, Dixon WG, Fu B, Ustianowski AP, et al. Anti-TNF therapy is associated with an increased risk of serious infections in patients with rheumatoid arthritis especially in the first 6 months of treatment: updated results from the British Society for Rheumatology Biologics Register with special emphasis on risks in the elderly. Rheumatology (Oxford, England). 2011;50(1):124-31. https://doi.org/10. 1093/rheumatology/keq242.
28. Singh JA, Wells GA, Christensen R, Tanjong Ghogomu E, Maxwell L, Macdonald JK, et al. Adverse effects of biologics: a network meta-analysis and Cochrane overview. Cochrane Database Syst Rev. 2011;2:Cd008794. https://doi.org/10.1002/ 14651858.cd008794.pub2.

29. Office for National Statistics. National life tables: United Kingdom (2013-2015). https://www.ons.gov.uk/ peoplepopulationandcommunity/birthsdeathsandmarriages/

lifeexpectancies/datasets/

nationallifetablesunitedkingdomreferencetables. Acccessed 23 May 2017.

30. Wong K, Gladman DD, Husted J, Long JA, Farewell VT. Mortality studies in psoriatic arthritis: results from a single outpatient clinic. I. Causes and risk of death. Arthritis Rheum. 1997;40(10):1868-72. https://doi.org/10.1002/15290131(199710)40:10<1868::AID-ART21>3.0.CO;2-W.

31. Personal Social Services Research Unit (PSSRU). Unit costs of health and social care (2016). http://www.pssru.ac.uk/projectpages/unit-costs/. Accessed 23 May 2017.

32. National Institute for Health and Care Excellence (NICE). PMG9: Guide to the methods of technology appraisal - the reference case (2013). https://www.nice.org.uk/process/pmg9/ chapter/the-reference-case. Accessed 23 May 2017.

33. Mease P, McInnes I, Kirkham B, Kavanaugh A, Rahman P. Secukinumab provides sustained improvements in psoriatic arthritis: 2-year efficacy and safety results from a Phase 3 randomized, double-blind, placebo-controlled trial. Arthritis Rheumatol. 2014;66:S423-4. 